\title{
SOME NEW GENERALIZATIONS OF WEIGHTED DYNAMIC HARDY-KNOPP TYPE INEQUALITIES WITH KERNELS
}

\author{
ESSAM AWWAD
}

Abstract. In this paper, we establish some new generalizations of weighted dynamic HardyKnopp type inequalities with kernels on time scales and also, some new characterizations of the weights for these inequalities in different spaces. The main results will be proved by using the Hölder inequality, the Jensen inequality and the Minkowski inequality. These inequalities (when $\mathbb{T}=\mathbb{R}$ ) contain the characterization of Kaijser, Nikolova, Persson and Wedestig. Also, (when $\mathbb{T}=\mathbb{N}$ ) our results are essentially new.

Mathematics subject classification (2020): 26D10, 26D15, 34N05.

Keywords and phrases: Hardy type inequality, Hardy-Knopp type inequalities, time scales, weighted functions, inequalities.

\section{REFERENCES}

[1] R. P. Agarwal, D. O'Regan, S. H. Saker, Dynamic Inequalities on Time Scales, Springer Cham Heidlelberg New York Dordrechet London 2014.

[2] R. P. Agarwal, D. O’Regan, S. H. Saker, Hardy Type Inequalities on Time Scales, Springer, 2016.

[3] R. Bibi, M. Bohner, J. PeČArić, S. VArošAnec, Minkowski and Beckenbach-Dresher inequalities and functionals on time scales, J. Math. Inequal. 7 (2013), no. 3, 299-312.

[4] M. Bohner, A. Peterson, Dynamic Equations on Time Scales: An Introduction with Applications, Birkhäuser, Boston, Mass, USA, 2001.

[5] M. Bohner, A. Peterson, Advances in Dynamic Equations on Time Scales, Birkhäuser, Boston, 2003.

[6] A. CižmeŠIJA, J. PeČArIĆ, L. E. Persson, On strengthened Hardy and Pólya-Knopp's inequalities, J. Approx. Theory 125, (2003), 74-84.

[7] E. T. Copson, Note on series of positive terms, J. Lond. Math. Soc. 3 (1927), 49-51.

[8] G. H. HARDY, Notes on a theorem of Hilbert, Math. Z. 6 (1920), 314-317.

[9] G. H. HARDY, Notes on some points in the integral calculus, LX. An inequality between integrals, Mess. Math. 54 (1925), 150-156.

[10] G. H. HARDY, J. E. LiTTLEWOOD, Elementary theorems concerning power series with positive coefficents and moment constants of positive functions, J. Für Math. 157 (1927), 141-158.

[11] G. H. HARDY, Notes on some points in the integral calculus (LXIV), Mess. Math. 57 (1928), 12-16.

[12] H. P. HeInIG, Weighted norm inequalities for certain integral operators II, Proc. Amer. Math. Soc. 95 (1985), no. 3, 387-395.

[13] H. Heinig, L. Maligranda, Weighted inequalities for monotone and concave functions, Studia Math. 116 (1995), 133-165.

[14] S. KaiJser, L. E. Persson, A. ÖBerg, On Carleman and Knopp's inequalities, J. Approx. Theory 117, (2002), 140-151.

[15] S. Kaijser, L. Nikolova, L. E. Persson, A. Wedestig, Hardy type inequalities via convexity, Math. Inequal. Appl. 8 (2005), no. 3, 403-417.

[16] K. KNopp, Über Reihen mit positiven Gliedern, J. London. Math. Soc. 3 (1928), 205-311.

[17] A. Kufner, L. E. Persson, Weighted Inequalities of Hardy Type, World Scientific Publishing, 2003. 
[18] A. Kufner, L. Maligranda, L. E. Persson, The Hardy Inequalities: About its History and Some Related Results, Vydavatelsk servis, 2007.

[19] L. LeINDLER, Generalization of inequalities of Hardy and Littlewood, Acta Sci. Math. (Szeged), 31 (1970), 285-297.

[20] B. Opic A. Kufner, Hardy-Type Inequalities, Pitman Research Notes in Mathematics Series, Longman Scientific and Technical, Harlow, 1990.

[21] U. M. ÖZKan, H. Yildirim, Hardy-Knopp type inequalities on time scales, Dyn. Syst. Appl. 17 (2008), 477-486.

[22] U. M. ÖZKan, H. YILdirim, Time scale Hardy-Knopp type integral inequalities, Communications in Mathematical Analysis, 6 (1), (2009).

[23] S. H. Saker, R. R. Mahmoud, A. Peterson, Weighted Hardy-type inequalities on time scales with applications, Mediterr. J. Math. 13 (2015), no. 2, 585-606.

[24] G. J. Sinnamon, Weighted Hardy, Opial-type inequalities, J. Math. Anal. Appl. 160 (1991), 434-445.

[25] V. D. STEPANOV, Boundedness of linear integral operators on a class of monotone functions, Siberian Math. J. 32 (1991), 540-542. 